\title{
Emotion Processing and its Relationship to Social Functioning in Schizophrenia Patients
}

\section{Citation}

Hooker, Christine, and Sohee Park. 2002. Emotion processing and its relationship to social functioning in schizophrenia patients. Psychiatry Research 112(1): 41-50.

\section{Published Version}

http://dx.doi.org/10.1016/S0165-1781(02)00177-4

\section{Permanent link}

http://nrs.harvard.edu/urn-3:HUL.InstRepos:3157891

\section{Terms of Use}

This article was downloaded from Harvard University's DASH repository, and is made available under the terms and conditions applicable to Other Posted Material, as set forth at http:// nrs.harvard.edu/urn-3:HUL.InstRepos:dash.current.terms-of-use\#LAA

\section{Share Your Story}

The Harvard community has made this article openly available.

Please share how this access benefits you. Submit a story.

\section{Accessibility}




\title{
Emotion processing and its relationship to social functioning in schizophrenia patients
}

\author{
Christine Hooker ${ }^{\mathrm{a}, *}$, Sohee Park ${ }^{\mathrm{b}}$ \\ ${ }^{a}$ Helen Wills Neuroscience Institute, 4143 Tolman Hall \#5050, University of California at Berkeley, Berkeley, CA 94720-5050, \\ USA \\ ${ }^{\mathrm{b}}$ Department of Psychology, Vanderbilt University, Wilson Hall, Nashville, TN 37240, USA
}

Received 12 April 2001; received in revised form 3 June 2002; accepted 22 July 2002

\begin{abstract}
Schizophrenia patients have demonstrated deficits in affect recognition. Whether this deficit is part of a general difficulty in face perception or a specific problem in affect recognition is debatable. However, there is little research investigating the functional consequences of difficulties in identifying emotion in schizophrenia patients. We tested 20 chronic, medicated schizophrenia patients and 27 normal control participants on a battery of face recognition and affect recognition tasks. A subset of 14 patients was rated on the Social Dysfunction Index. Results demonstrated that schizophrenia patients were less accurate than normal control participants on face recognition, facial affect recognition and vocal affect recognition tasks, but among schizophrenia patients, only affect recognition performance was related to social functioning. These results suggest that schizophrenia patients have general face processing deficits, but affect recognition deficits may lead to more problems in social behavior.
\end{abstract}

(c) 2002 Elsevier Science Ireland Ltd. All rights reserved.

Keywords: Affect recognition; Face perception; Prosody; Social Dysfunction Index; Non-verbal communication

\section{Introduction}

There has been a considerable amount of research in the past 20 years investigating schizophrenia patients' ability to recognize emotional expression in others. Most of these investigations have found that schizophrenia patients have defi-

\footnotetext{
*Corresponding author.

E-mail address:

chooker@socrates.berkeley.edu (C. Hooker),

sohee.park@vanderbilt.edu (S. Park).
}

cits in both facial and vocal affect recognition (e.g. Archer et al., 1994; Baudouin et al., 2002; Borod et al., 1993; Edwards et al., 2001; Feinberg et al., 1986; Heimberg et al., 1992; Mandal et al., 1998; Penn et al., 2000; Schneider et al., 1995; Walker et al., 1984), and that these deficits are not related to age, gender, medication status or neuroleptic dose (Kline et al., 1992; Poole et al., 2000; Salem et al., 1996; Schneider et al., 1995).

Discussion in this literature has focused on whether the schizophrenia patients' poor perform- 
ance on affect recognition tasks is a differential deficit in affect recognition, a general face or voice perception deficit, or part of global cognitive impairment (Chapman and Chapman, 1978). Most studies that have used a face perception control task as compared to a face affect recognition task have found that schizophrenia patients perform more poorly than normal control participants on both facial affect recognition and the face perception control task, indicating that schizophrenia patients have a general impairment in face perception (e.g. Kerr and Neale, 1993). Investigations of voice affect recognition have been less consistent. Several studies have shown a specific deficit for schizophrenia patients in affective prosody as compared to a non-affective prosody control task (e.g. Murphy and Cutting, 1990), but others have demonstrated that affective prosody is part of a general deficit in analyzing both emotional and nonemotional vocal cues (e.g. Kerr and Neale, 1993).

Investigations of the relationship between affect recognition and specific symptoms have yielded mixed findings. Most studies point to a relationship between face affect recognition and negative symptoms, but the specificity of this relationship is still unclear. Several studies have found general face processing deficits (both affect and identity recognition) related to specific negative symptoms, such as anergia (Lukoff et al., 1986; Mueser et al., 1996), whereas other studies have found that facial affect recognition deficits but not face perception correlated with specific negative symptoms (e.g. alogia) (Gaebel and Wölwer 1992; Kohler et al., 2000) and overall severity of negative symptoms (Baudouin et al., 2002; Schneider et al., 1995; Lewis and Garver, 1995). However, some of these studies also found a specific relationship between affect recognition and positive symptoms such as bizarre behavior (Schneider et al., 1995), thought disorder (Kohler et al., 2000), and overall positive symptoms (Lewis and Garver, 1995). One study reported that combined voice and face affect recognition scores were related to positive and disorganized symptoms but not negative symptoms (Poole et al., 2000), and several groups reported no significant correlations between affect recognition performance and clinical symptoms (Salem et al., 1996; Borod et al., 1993; Muzekari and Bates,
1977; Novic et al., 1984; Wölwer et al., 1996; Addington and Addington, 1998).

In addition, researchers have investigated whether affect recognition deficits might be related to certain stages or characteristics of schizophrenic illness (Mueser et al., 1996). For example, a recent study reported that schizophrenia patients in acute stages of illness demonstrated a specific affect recognition deficit, but chronic schizophrenia patients demonstrated a general face processing deficit (Penn et al., 2000). However, others who have investigated stages and characteristics of schizophrenic illness such as number of hospitalizations, duration of hospital stay, and chronicity of illness have found no specific association with affect recognition abilities (Salem et al., 1996; Addington and Addington, 1998).

Though it has been difficult to pin down symptom correlates of affect recognition, evidence demonstrates that affect recognition deficits, as well as face processing deficits, are a stable feature of schizophrenia pathology (Addington and Addington, 1998). Furthermore, face perception and face affect recognition deficits have also been shown in schizotypal personality disorder patients (Mikhailova et al., 1996), and schizotypic college students (Poreh et al., 1994). Clearly, schizophrenia and schizophrenia spectrum patients have problems with this process that may lead to specific consequences.

Thus, an important question is-what are the functional consequences of emotion recognition deficits for schizophrenia patients? And furthermore-do emotion recognition deficits have different or more specific consequences in daily functioning than the general cognitive impairments that are associated with schizophrenic illness?

There have only been a few studies specifically addressing the relationship of affect recognition and social functioning. Mueser et al. (1996) tested chronic, medicated inpatients with two measures of social competence: a conversation probe, which was rated for specific elements of social competence, and the Social Behavior Schedule (SBS), which included four areas of social behavior: social mixing, inappropriate behavior, personal appearance and hygiene, and activity level. They found a correlation between a composite of social com- 
petence and overall face perception performance. Interestingly, both facial affect recognition and facial effect recognition were associated with social functioning as rated by the SBS, but only facial affect recognition deficits were associated with the conversation probe. Another study of ward behavior found that facial affect recognition deficits were associated with reduced social competence, social interest, and hygiene, even after controlling for other cognitive abilities (Penn et al., 1996). Poole et al. (2000) measured both facial and vocal affect recognition in outpatient schizophrenia patients and found that even when partialing out nonemotional cognitive ability, affect recognition was significantly correlated with disorganized symptoms from the Positive and Negative Syndrome Scale (PANSS) and interpersonal relationships as measured by the Quality of Life Scale.

Our study sought to investigate further the relationship between affect recognition and social functioning by using both vocal and facial affect recognition tasks and a different social functioning measure than has been used in the past, the Social Dysfunction Index (SDI) (Munroe-Blum et al., 1996). This measure has nine subscales assessing a wide range of social behavior. In particular, whereas previous measures have embedded communication problems into other measures of functioning-such as relationship problems-this measure has a specific communication subscale, which we believed would be sensitive to affect recognition problems. We predicted that schizophrenia patients would have deficits in both affective and nonaffective tasks but that only deficits in affect recognition would predict social dysfunction. We specifically identified communication dysfunction as a domain of social functioning that would be particularly sensitive to affect recognition deficits.

\section{Methods}

\subsection{Participants}

Twenty schizophrenia patients were recruited from a local residential mental health care facility. The schizophrenia patients had a mean age of 39.3
(S.D. $=8.5)$ and a mean education of 12.7 years $($ S.D. $=2.9)$. Patients had an average duration of illness of approximately 18.8 years (S.D. $=10.2$ ). There were 15 males and 5 females in our sample. Fifteen of the patients were Caucasian. All of the patients were taking antipsychotic medication at the time of testing with a mean chlorpromazine equivalent dose of approximately $1043 \mathrm{mg}$. Advanced clinical graduate students under the supervision of a licensed psychologist diagnosed schizophrenia patients according to DSM-IV criteria. Information about the patient's illness was acquired through the Schedule for Affective Disorders and Schizophrenia (SADS) interview and medical chart reviews (Endicott and Spitzer, 1978). Exclusion criteria were comorbid Axis I disorders, neurological disorders, substance abuse, history of severe head injury, or over 55 years of age.

Twenty-seven normal control participants were recruited via postings in the community. There were 14 males and 13 females in our sample. Nineteen of the participants were Caucasian. The mean age of the normal participants was 34.4 $($ S.D. $=8.3)$, and they had a mean level of education of 13.6 years $($ S.D. $=1.8)$. Participants were screened for history of psychiatric disturbance, substance abuse, neurological disorders, possible schizotypal personality [i.e. scoring above 45 on the Schizotypal Personality Questionnaire (Raine, 1991)]. Schizophrenia patients were slightly older than the normal control participants, but the age difference was not significant $\left(F_{1,45}=3.8, P>\right.$ $0.05)$. There was no significant difference in years of education $\left(F_{1,45}=1.6, P>0.1\right)$ between the two groups. All participants gave informed consent and were paid for their participation.

\subsection{Design and procedure}

All participants were tested on five experimental tasks given in a counterbalanced order across subjects:

\subsubsection{Test of Facial Recognition (Benton et al., 1983)}

Participants are shown a face and asked to identify one or three pictures of that same person among six choices. The faces in the answer choices 
are shown with varying facial profiles and light conditions. There are 22 stimulus pictures and 54 matches in the test (i.e. 54 total items). The exam is administered in a booklet form and there is no time constraint. The internal-consistency reliability (Cronbach's alpha) for the Test of Facial Recognition for this sample was alpha $=0.71$.

\subsubsection{Facial Affect Recognition}

We constructed this task to have approximately the same task demands as the Test of Facial Recognition but with emotion recognition as the tested domain. Participants are shown a stimulus picture and asked to match the displayed emotion with the same emotion portrayed in one of the picture choices. There are seven picture choices, each displaying a different emotion (happy, angry, sad, disgust, fear, surprise, or contempt). The person shown in the stimulus face is not represented in the answer choices. The test is administered in booklet form. Stimuli photographs are taken from a standardized set of stimulus faces (Biehl et al., 1997). The test has 28 items. Cronbach's alpha for the Facial Affect Recognition task for this sample was alpha $=0.79$.

\subsubsection{Neutral Face Recognition}

The Neutral Face Recognition task design is identical to the Facial Affect Recognition task except that the faces have a neutral expression. Participants are shown a face and asked to match it to another picture of the same face out of seven choices. This task provides a baseline control for the Facial Affect Recognition task since the identities and visual properties of the stimulus photographs are exactly the same. The test has 28 items and is administered in booklet form. Cronbach's alpha for the Neutral Face Recognition task in this sample was alpha $=0.89$.

The stimuli from the Neutral Face Recognition and Facial Affect Recognition tasks were taken from the Japanese and Caucasian Facial Expression of Emotion (JACFEE) and Neutral Faces (JACNeuF) series constructed by Paul Ekman and David Matsumoto. There has been extensive previous work documenting the reliability and validity of these stimuli (Biehl et al., 1997).

\subsubsection{Vocal Affect Recognition}

This task was taken from the Diagnostic Analysis of Nonverbal Accuracy (DANVA2) (Nowicki and Duke, 1994). Participants listen to a tape recording of a person saying a single sentence with different emotional intonation and are asked to identify what the person is feeling by circling one of four choices listed on an answer sheet (happy, sad, angry, and fearful). Half of the 24 items are identified as high emotional intensity and the other half as low emotional intensity. The test has been shown to be reliable and valid (Baum and Nowicki, 1998). Cronbach's alpha for the Vocal Affect Recognition task for this sample was alpha $=0.69$.

\subsubsection{Pitch Perception}

In order to ascertain that all participants are able to discriminate differences in pitch that may contribute to prosodic analysis, we developed a Pitch Perception task in which participants listen to an example of a high and low tone and then identify whether each successive tone is high or low. The test has 10 items.

\subsubsection{Social functioning}

In addition to the experimental tasks, 14 schizophrenia patients ${ }^{1}$ were rated on the SDI (see Munroe-Blum et al. (1996) for psychometric properties). The SDI was created specifically to assess social functioning in schizophrenia and other major psychiatric disorders. It assesses functioning in the following different domains: (A) Public Self (behavior, appearance, social presentation); (B) Independent Living; (C) Occupational Functioning; (D) Family Relationships; (E) Important Relationships other than family; (F) Community/ Leisure/Recreation; (G) Acceptance and Adherence to Health Regimens; (H) Communication; (I) Locus of Control; Total SDI Rating.

The head caseworker of the residential mental health facility was trained to use the scale and then rated each of the participants in the study. (The caseworker was blind to the purpose of the

\footnotetext{
${ }^{1}$ We were not able to recruit all of our patients for this part of the study due to time constraints of the head case worker and because several patients were no longer at the mental health facility.
} 
Table 1

Performance and mean comparisons on the face tasks for the two groups

\begin{tabular}{|c|c|c|c|c|}
\hline Task & $\begin{array}{l}\text { Schizophrenia } \\
\text { patients }\end{array}$ & $\begin{array}{l}\text { Normal } \\
\text { controls }\end{array}$ & $t(45)$ & $P$ value \\
\hline Neutral Face Recognition & $97.3(7.5)$ & $100(0)$ & -1.9 & 0.07 \\
\hline Facial Affect Recognition & $77.1(16.6)$ & $89.8(10.4)$ & -3.2 & $0.003^{*}$ \\
\hline Test of Facial Recognition & $78.9(10.7)$ & $86.6(4.9)$ & -3.3 & $0.002^{*}$ \\
\hline
\end{tabular}

Performance measures are shown in percent correct.

* Statistically significant with Bonferroni-corrected $P$ value of 0.0167 .

study at the time of the rating.) The SDI is a semistructured interview assessing the past month of functioning. There are nine domains assessed and each domain is given three different scores on a scale of $0-3$ ( 0 , no dysfunction and 3 , major dysfunction): (1) dysfunction in the past month; (2) number of areas of dysfunction; and (3) satisfaction with level of functioning. Dysfunction and number of area scores are averaged for a domain score. All domains are combined for a Total SDI score. Higher scores indicate more dysfunction.

The SDI has been shown to be significantly correlated with other well-established scales of social functioning such as the Social Adjustment Scale (SAS-II) (Schooler et al., 1979), the Global Assessment Scale (GAS) (Endicott et al., 1976), and the Social Behavior Adjustment Scale (SBAS) (Platt et al., 1980). The SDI was also found to have high reliability, high predictive validity and to be sensitive to change (Munroe-Blum et al., 1996).

\section{Results}

The visual and auditory tasks were analyzed separately.

\subsection{Visual Face tasks}

Table 1 shows results of a series of independent $t$-tests with performance on each of the three face tasks (Neutral Face Recognition, Facial Affect Recognition, and Test of Facial Recognition) as the dependent variable and diagnostic group as the independent variable. There was no significant difference (two-tailed test, Bonferroni adjusted $P$ value of 0.0167 ) between schizophrenia patients and normal control participants on the Neutral Face Recognition task, but there was a significant difference between the two subject groups on the Test of Facial Recognition and the Facial Affect Recognition task, with schizophrenia patients performing worse than normal controls on both tests. In other words, schizophrenia patients performed as well as control participants on a simple control task, but they did not show a specific deficit on the Facial Affect Recognition task when compared to an equally difficult facial identity recognition task.

A repeated measures ANOVA on the Facial Affect Recognition task with accuracy for emotion type (happy, sad, angry, fearful, surprise, disgust, and contempt) as the dependent variable and diagnostic group as the independent variable $(P<0.05$, 2-tailed) did not show any particular pattern of errors for schizophrenia patients relative to controls (Emotion errors $\times$ Diagnosis interaction $\left(F_{6,270}=\right.$ $1.1, P>0.1))$. That is, schizophrenia patients were not making an excess number of errors for a particular emotion, and there was no evidence of bias in the error pattern. There was also no effect for the race or gender of the person in the stimuli picture for either participant group. A repeated measures ANOVA on the Facial Affect Recognition task using gender and race as the dependent variables and diagnostic group as the independent variable $(P<0.05,2$-tailed $)$ showed no significant difference in the ability to match emotional expression for Japanese as compared to Caucasian faces $\left(F_{1,45}=0.383, P>0.1\right)$ or male faces as compared to female faces $\left(F_{1,45}=2.0, P>0.1\right)$.

\subsection{Auditory tasks}

Table 2 shows performance measures for the auditory tasks. Both groups performed at $100 \%$ 
accuracy for the Pitch Perception task, so no further analysis was done on this measure. Independent $t$-tests (two-tailed test, Bonferroni-adjusted $P$ value of 0.0167 ) were used to investigate the difference in performance accuracy between the two participant groups on the Vocal Affect Recognition task total score as well as scores for high and low emotion stimuli within that test. These results showed that schizophrenia patients performed significantly worse than normal control participants on each of these measures of Vocal Affect Recognition. To further investigate the effect of emotional intensity on performance, we computed a repeated measures ANOVA with accuracy on high and low intensity items as the dependent variable and diagnostic group as the independent variable. This analysis (Fig. 1) revealed a significant interaction $(P<0.05$, twotailed) between emotional intensity and diagnosis $\left(F_{1,43}=7.2, P=0.01\right)$, such that although both schizophrenia patients and normal controls had more difficulty with low intensity stimuli, the low intensity stimuli were more problematic for the schizophrenia patients than for the controls.

\subsection{Social functioning}

Fourteen of the schizophrenia patients were rated on the Social Dysfunction Scale (SDI). As mentioned above, the scale has a total dysfunction score as well as nine specific domain scores. Each of these scores (Total SDI and the nine domain scores) was entered into a correlation matrix to investigate the relationship between social functioning and our cognitive measures of face perception and of face and vocal affect recognition. This analysis revealed no significant (two-tailed, $P<$

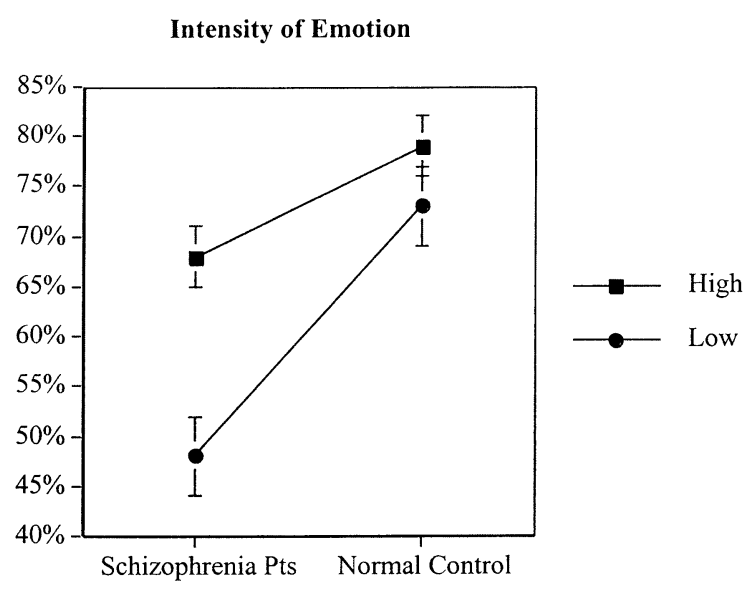

Fig. 1. Effect of intensity of emotion on performance in the Vocal Affect Recognition task.

$0.05)$ relationship between the Total SDI score and any of the experimental measures. However, there were significant correlations with specific domain scores (Table 3), such that higher accuracy on the affective tasks predicted less dysfunction in specific areas. Performance on the Facial Affect Recognition task was negatively correlated with communication dysfunction and occupation dysfunction. In addition, performance on the Facial Affect Recognition task was negatively correlated with problems in public appearance and behavior at the trend level (two-tailed, $P<0.1$ ). Performance on Vocal Affect Recognition was negatively correlated with occupational dysfunction. Performance on the Test of Facial Recognition did not correlate in the predicted direction with any of the social dysfunction domains.

Table 2

Performance and mean comparisons on the auditory tasks for the two groups

\begin{tabular}{|c|c|c|c|c|}
\hline Task & $\begin{array}{l}\text { Schizophrenia } \\
\text { patients }\end{array}$ & $\begin{array}{l}\text { Normal } \\
\text { controls }\end{array}$ & $t(45)$ & $P$ value \\
\hline Pitch Perception & $100(0)$ & $100(0)$ & $\mathrm{N} / \mathrm{A}$ & $\mathrm{N} / \mathrm{A}$ \\
\hline Vocal Affect Recognition-total & $59.0(13.2)$ & $75.0(12.1)$ & -4.3 & $<0.0001^{*}$ \\
\hline Vocal Affect—high intensity & $67.9(14.4)$ & $78.6(11.7)$ & -2.7 & $0.01^{*}$ \\
\hline Vocal Affect-low intensity & $48.3(15.7)$ & $72.5(11.7)$ & -4.7 & $<0.0001^{*}$ \\
\hline
\end{tabular}

Performance measures are shown in percent correct.

* Significant at Bonferroni-corrected $P$ value of 0.0167 . 
Table 3

The correlation matrix for the cognitive tasks and specific domains of social functioning

\begin{tabular}{lccr}
\hline Task & $\begin{array}{l}\text { Face } \\
\text { Affect }\end{array}$ & $\begin{array}{l}\text { Vocal } \\
\text { Affect }\end{array}$ & $\begin{array}{l}\text { Test of Facial } \\
\text { recognition }\end{array}$ \\
\hline Face Affect Recognition & & & 0.35 \\
Vocal Affect Recognition & $0.43^{*}$ & $0.47^{*}$ & -0.1 \\
Test of Facial Recognition & $-0.59^{* *}$ & $-0.58^{* *}$ & -0.1 \\
Communication dysfunction & $-0.56^{* *}$ & -0.1 & -0.04 \\
Occupational dysfunction & $-0.46^{*}$ & \\
Public Self & & \\
\hline
\end{tabular}

${ }^{*} P<0.1$ (2-tailed, unadjusted).

${ }^{* *} P<0.05$ (2-tailed, unadjusted).

\section{Discussion}

We examined visual and auditory affect recognition abilities and their relationship to social functioning in schizophrenia patients. Our results confirm and extend earlier findings that affect recognition is a problem for schizophrenia patients and is related to specific domains of social functioning.

In our cognitive tasks, we found that schizophrenia patients had deficits in face recognition, facial affect recognition, and vocal affect recognition, compared to normal control participants. In the analysis of our auditory tasks, although we were unable to conclude that vocal affect recognition is a differential deficit (Chapman and Chapman, 1978), it was clear that difficulties in vocal affect recognition were not related to basic perceptual difficulties. In addition, there was an interaction within the vocal affect recognition task such that schizophrenia patients were much less accurate on items with a low emotional intensity. This fits with other findings that if there is any ambiguity in the social situation, schizophrenia patients are more likely to make an erroneous interpretation (Corrigan and Nelson, 1998).

In the analysis of our three face tasks, we replicated earlier results that schizophrenia patients have difficulties with face perception, in general, as well as facial affect recognition (Kerr and Neale, 1993; Mueser et al., 1996, 1997; Salem et al., 1996). The performance of the schizophrenia patients and the normal control participants in our sample was roughly equivalent to scores found for the Test of Facial Recognition and similar facial affect recognition and discrimination tasks in other studies (e.g. Salem et al., 1996; Kerr and Neale, 1993; Mueser et al., 1996; Addington and Addington, 1998; Borod et al., 1993; Bellack et al., 1996). In most previous studies, schizophrenia patients performed worse than normal control participants on both affective and non-affective face tasks, but the task by diagnosis interaction supporting a differential deficit was not significant (e.g. Salem et al., 1996; Kerr and Neale, 1993). However, it is interesting that the majority of studies (e.g. Kerr and Neale, 1993; Addington and Addington, 1998; Borod et al., 1993) found the greatest difference between schizophrenia and normal performance on the emotion tasks, not face recognition. This suggests that perhaps there is a differential deficit but no single study has enough statistical power to show the effect. A comprehensive meta-analysis comparing performance on face perception and face affect perception would offer more decisive results on this issue.

We found the Test of Facial Recognition and the Facial Affect Recognition task to be correlated at the trend level $(r=0.43, P<0.1)$ among the schizophrenia patients but found no significant correlation between these tests in the control participants. This result is consistent with other studies showing significant correlations among the patient group (Addington and Addington, 1998; Salem et al., 1996; Kerr and Neale, 1993; Mueser et al., 1996). However, there are several investigations that reported no correlation between face affect and face recognition (Borod et al., 1993; Poole et al., 2000). Given the inconclusive results across studies, it seems the relationship between facial 
affect recognition and face perception needs further refinement.

Accurate performance on the Facial Affect Recognition task was significantly related to less dysfunction in communication, occupation, and, at the trend level, self-presentation. However, the non-affective Test of Facial Recognition was not significantly related to any of the social functioning domains.

Since successful communication requires understanding nonverbal cues, we hypothesized that communication would be affected by problems in affect recognition and that is what we found. This finding fits with Mueser et al. (1996) who found that face affect recognition but not non-affective face recognition predicted successful communication during a 3-5-min conversation.

It follows logically that a person who is having difficulty with communication may also have difficulty forming or maintaining close relationships. For example, Poole et al. (2000) found a significant relationship between affect recognition and interpersonal relations. Although there are two domains assessing interpersonal relationships with the SDI (Family Relationships and Important Relationships other than Family), affect recognition was not a significant predictor for functioning in either of these domains. However, successful relationships with family and friends incorporate other variables (such as desire to maintain relationships and feelings of attachment) in addition to immediate communication skills. Because the SDI separates friendships, family relationships and communication, the communication domain relates most specifically to the ability to understand and identify nonverbal cues.

Interestingly, Poole et al. (2000) did not find any correlation between affect recognition skills and occupational functioning whereas this correlation was significant in our study for both face and vocal affect recognition. It is unclear why this may be the case. One possibility is that since our participants were living in a residential facility and working in sponsored job programs, their occupational functioning was closely monitored. Thus, our measure may have been more sensitive to a full range of problems on the job that may not be discovered by self-report or interview.
The Public Self domain on the SDI assesses dysfunction by taking into account odd behavior or dress, number of times the patient has caused disturbances or been barred from public places, etc., and this domain was correlated with facial affect recognition at the trend level. This result fits with Poole et al. (2000) who reported a significant correlation between affect recognition abilities and bizarre behavior and appearance, even after controlling for intellectual and clinical variables. Mueser et al. (1996) also found a significant relationship between Facial Affect Recognition and a personal appearance measure of the SBS.

Although our facial and vocal affect recognition tasks were positively associated, the correlation between them $(r=0.35, P>0.1)$ was not statistically significant. Most reports of vocal and facial affect recognition show a significant correlation across modalities (e.g. Kerr and Neale, 1993; Poole et al., 2000). However, our two affective tasks may have required different cognitive skills. The Vocal Affect Recognition task required subjects to identify the emotion by choosing one of four labeled emotions, whereas the Facial Affect Recognition task required subjects to match a picture of a target emotion to one of seven possible choices. In other words, in addition to assessing a wider range of emotions, the face emotion task had no verbal component, so participants could match the emotion on perceptual features alone.

Accurate performance on the Vocal Affect Recognition task was significantly related to less dysfunction in occupational performance, but it was not significantly related to level of dysfunction in communication or self-presentation. We had hypothesized that vocal affect recognition would be a strong predictor of communication skills so this result was surprising. However, it could be that since our face emotion task was purely perceptual, had no time constraints and covered a wider range of emotion, it was a better estimate nonverbal decoding ability.

Overall our results show that face and voice affect recognition is a significant predictor of specific domains of social functioning. This confirms and extends results from other laboratories (Mueser et al., 1996; Penn et al., 1996; Poole et al., 2000) and adds evidence to the theory that 
deficits in reading nonverbal cues may be a contributing factor in the well-documented social problems of schizophrenia patients (Bellack et al., 1996; Penn et al., 2001; Wallace, 1984). Although the direction of causation is still unclear, the accumulation of evidence illustrating an association between affect recognition and social functioning may inform research in effective rehabilitation and social skills training. Future research might also investigate neurobiological factors underlying these deficits.

\section{Acknowledgments}

This work was supported in part by NARSAD and NIMH. We thank Jennifer Ochs, Zubair Tajuddin, Karen Madda, and Michelle Pagel for their help in data collection and subject recruitment.

\section{References}

Addington, J., Addington, D., 1998. Facial affect recognition and information processing in schizophrenia and bipolar disorder. Schizophrenia Research 32, 171-181.

Archer, J., Hay, D.C., Young, A.W., 1994. Movement, face processing, and schizophrenia: evidence of a differential deficit in expression analysis. British Journal of Clinical Psychology 33, 517-528.

Baudouin, J.-Y., Martin, F., Tiberghien, G., Verlut, I., Frank, N., 2002. Selective attention to facial emotion and identity in schizophrenia. Neuropsychologia 40, 503-511.

Baum, K.M., Nowicki, S.N., 1998. Perception of emotion: measuring decoding accuracy of adult prosodic cues varying in intensity. Journal of Nonverbal Behavior 22, 89-106.

Bellack, A.S., Blanchard, J.J., Mueser, K.T., 1996. Cue availability and affect perception in schizophrenia. Schizophrenia Bulletin 22, 535-544.

Benton, A.L., Hamsher, K. de S., Varney, N.R., Spreen, O., 1983. Contributions to Neuropsychological Assessment. Oxford University Press, New York.

Biehl, M., Matsumoto, D., Ekman, P., Hearn, V., 1997. Matsumoto and Ekman's Japanese and Caucasian Facial Expression and Emotion (JACFEE): reliability data and cross national differences. Journal of Nonverbal Behavior 21, 3-21.

Borod, J.C., Martin, C.C., Alpert, M., Brozgold, A., Welkowitz, J., 1993. Perception of facial emotion in schizophrenia and right brain damaged patients. Journal of Nervous and Mental Disease 181, 494-502.

Chapman, L., Chapman, J., 1978. The measurement of differential deficit. Journal of Psychiatric Research 14, 303-311.

Corrigan, P.W., Nelson, D.R., 1998. Factors that affect social cue recognition in schizophrenia. Psychiatry Research 78, 189-196.
Edwards, J., Pattison, P.E., Jackson, H.J., Wales, R.J., 2001. Facial affect and affective prosody recognition in firstepisode schizophrenia. Schizophrenia Research 48, 235-253.

Endicott, J., Spitzer, R.L., 1978. A diagnostic interview: the schedule for Affective Disorders and Schizophrenia. Archives of General Psychiatry 35, 837-844.

Endicott, J., Spitzer, R.L., Fleiss, J.F., Cohen, J., 1976. The Global Assessment Scale: a procedure for measuring overall severity of psychiatric disturbance. Archives of General Psychiatry 33, 766-771.

Feinberg, T.E., Rifkin, A., Schaeffer, C., Walker, E., 1986. Facial discrimination and emotional recognition in schizophrenia and emotional disorders. Archives of General Psychiatry 43, 276-279.

Gaebel, W., Wölwer, W., 1992. Facial expression and emotional face recognition in schizophrenia and depression. European Archives of Psychiatry and Clinical Neuroscience 242, 46-52.

Heimberg, C., Gur, R.E., Erwin, R.J., Shtatsel, D.L., Gur, R.C., 1992. Facial emotion discrimination: III. Behavioral findings in schizophrenia. Psychiatry Research 42, 253-265.

Kerr, S.L., Neale, J.M., 1993. Emotion perception in schizophrenia: specific deficit or further evidence of generalized poor performance. Journal of Abnormal Psychology 102, 312-318.

Kline, J.S., Smith, J.E., Ellis, H.C., 1992. Paranoid and nonparanoid schizophrenic processing of facially displayed affect. Journal of Psychiatric Research 26, 169-182.

Kohler, C.G., Bilker, W., Hagendoorn, M., Gur, R.E., Gur, R.C., 2000. Emotion recognition deficit in schizophrenia: association with symptomatology. Biological Psychiatry 48, 127-136.

Lewis, S.F., Garver, D.L., 1995. Treatment and diagnostic subtype in facial affect recognition in schizophrenia. Journal of Psychiatric Research 29, 5-11.

Lukoff, D., Liberman, R.P., Nuechterlein, K.H., 1986. Symptom monitoring in the rehabilitation of schizophrenic patients. Schizophrenia Bulletin 12, 578-603.

Mandal, M.K., Pandey, R., Prasad, A.B., 1998. Facial expressions of emotions and schizophrenia. Schizophrenia Bulletin 24, 399-412.

Mikhailova, E.S., Vladimirova, T.V., Iznak, A.F., Tsusulkovskaya, E.J., Sushko, N.V., 1996. Abnormal recognition of facial expression of emotions in depressed patients with major depression disorder and schizotypal personality disorder. Biological Psychiatry 40, 697-705.

Mueser, K.T., Doonan, R., Penn, D.L., Blanchard, J.L., Bellack, A.S., Nishith, P., de Leon, J., 1996. Emotion recognition and social competence in chronic schizophrenia. Journal of Abnormal Psychology 105, 271-275.

Mueser, K.T., Penn, D.L., Blanchard, J.L., Bellack, A.S., 1997. Affect recognition in schizophrenia: a synthesis of findings across three studies. Psychiatry 60, 301-308.

Munroe-Blum, H., Collins, E., McCleary, L., Nutall, S., 1996. The social dysfunction index for patients with schizophrenia and related disorders. Schizophrenia Research 20, 211-219. 
Murphy, D., Cutting, J., 1990. Prosodic comprehension and expression in schizophrenia. Journal of Neurology, Neurosurgery and Psychiatry 53, 727-730.

Muzekari, L.H., Bates, M.E., 1977. Judgement of emotion among chronic schizophrenics. Journal of Clinical Psychology 32, 662-666.

Nowicki, S., Duke, M.P., 1994. Individual differences in the nonverbal communication of affect: the diagnostic analysis of nonverbal accuracy scale. Journal of Nonverbal Behavior 18, 9-35.

Novic, J., Luchins, D.J., Perline, R., 1984. Facial affect recognition in schizophrenia: is there a differential deficit? British Journal of Psychiatry 144, 533-537.

Penn, D.L., Combs, D., Mohamed, S., 2001. Social cognition and social functioning in schizophrenia. In: Corrigan, P.W., Penn, D.L. (Eds.), Social Cognition and Schizophrenia. American Psychological Association, Washington, DC, pp. 97-121.

Penn, D.L., Combs, D.R., Ritchie, M., Francis, J., Morris, S., Townsend, M., 2000. Emotion recognition in schizophrenia: further investigation of generalized versus specific deficit models. Journal of Abnormal Psychology 109, 512-516.

Penn, D.L., Spaulding, W., Reed, D., Sullivan, M., 1996. The relationship of social cognition to ward behavior in chronic schizophrenia. Schizophrenia Research 20, 327-335.

Platt, S., Weyman, A., Hirsch, S., Hewett, S., 1980. The Social Behavior Assessment Schedule (SBAS): rationale, contents, scoring and reliability of a new interview schedule. Social Psychiatry 15, 43-55.

Poole, J.H., Tobias, F., Vinogradov, S., 2000. The functional relevance of affect recognition errors in schizophrenia.
Journal of the International Neuropsychological Society 6, 649-658.

Poreh, A.M., Whitman, R.D., Weber, M., Ross, T., 1994. Facial recognition in hypothetically schizotypal college students: the role of generalized poor performance. Journal of Nervous and Mental Disease 182, 503-507.

Raine, A., 1991. The SPQ: a scale for the assessment of schizotypal personality based on DSM-III-R criteria. Schizophrenia Bulletin 17, 555-564.

Salem, J.E., Kring, A.M., Kerr, S.L., 1996. More evidence for generalized poor performance in facial emotion perception in schizophrenia. Journal of Abnormal Psychology 105, 480-483.

Schneider, F., Gur, R.C., Gur, R.E., Shtatsel, D.L., 1995. Emotional processing in schizophrenia: neurobehavioral probes in relation to psychopathology. Schizophrenia Research 17, 67-75.

Schooler, N., Hogarty, G.E., Weissman, M.M., 1979. Social Adjustment Scale (II). In: Hargreaves, W.A., Atkinson, C.C., Sorenson, J.E. (Eds.), Resource Materials for Community Mental Health Program Evaluation. Superintendant of Documents, Government Printing Office, Washington, D.C. DHEW No. 79, p. 328.

Walker, E., McGuire, M., Bettes, B., 1984. Recognition and identification of facial stimuli by schizophrenics and patients with affective disorders. British Journal of Clinical Psychology $23,37-44$.

Wallace, C.J., 1984. Community and interpersonal functioning in the course of schizophrenic disorders. Schizophrenia Bulletin 10, 233-257.

Wölwer, W., Streit, M., Polzer, U., Gaebel, G., 1996. Facial affect recognition in the course of schizophrenia. European Archives of Psychiatry and Clinical Neuroscience 246, 165-170. 